Article

\title{
Separation of Microparticles from Suspension Utilizing Ultrasonic Standing Waves in a Piezoelectric Cylinder Actuator
}

\author{
Vytautas Ostasevicius ${ }^{1, *}$, Vytautas Jurenas ${ }^{1}$, Ievgeniia Golinka ${ }^{1}{ }^{(\mathbb{D})}$, Rimvydas Gaidys ${ }^{2}$ and \\ Algiment Aleksa ${ }^{3}$ \\ 1 Institute of Mechatronics, Kaunas University of Technology, LT51424 Kaunas, Lithuania; \\ vytautas.jurenas@ktu.lt (V.J.); ievgeniia.golinka@ktu.edu (I.G.) \\ 2 Department of Mechanical Engineering, Kaunas University of Technology, LT51424 Kaunas, Lithuania; \\ rimvydas.gaidys@ktu.lt \\ 3 Department of Mathematical Modeling, Kaunas University of Technology, LT51424 Kaunas, Lithuania; \\ algiment.aleksa@ktu.edu \\ * Correspondence: vytautas.ostasevicius@ktu.lt
}

Received: 30 January 2018; Accepted: 29 March 2018; Published: 31 March 2018

\begin{abstract}
A method of microparticle separation from larger volumes of suspension is proposed. A piezoelectric cylinder is selected as an ultrasonic wave actuator, the diameter and length of which the volume of the suspension to be purified depends. Numerically and experimentally, it is demonstrated that the low-level pressure field nodal circles of ultrasonic radiation standing waves concentrate microparticles at different velocities depending on the fluid viscosity. Numerical mathematical modeling has allowed us to identify the basic dynamic characteristics of the piezoelectric actuator to ensure a more effective process of microparticle separation. An important feature of the proposed method is that the ultrasonic radiation stresses that are directly applicable to cell membranes are inadequate to cause them damage.
\end{abstract}

Keywords: piezoelectric actuator; ultrasonic standing waves; mathematical model; 3D experimental setup

\section{Introduction}

Acoustic standing wave technology creates new opportunities to improve advanced particle and cell separating systems. The influence of ultrasonic waves on the diffracting phase is interesting itself as one of the fundamental physics effects of ultrasound. The work in [1] deals with the problem of ultrasound in the context of biomedical and clinical applications. It is suggested that only radiation pressure should be taken into account as a mechanism of drifting. Apart from the acoustic radiation forces, Rayleigh streaming flows are normally detected in acoustic-fluidic manipulation and sorting devices [2]. Several experimental studies were performed [3] to examine the ways bio-particles can be manipulated using acoustics. For the evaluation of the effectiveness of this technology, digital particle image velocimetry is an optical method of flow visualization, which is described in terms of linear system theory $[4,5]$, and the conditions for correlation analysis as a valid interrogation method are determined.

Continuous flow acoustic standing wave separation of particles in microscale systems was described in detail in [6-11]. A new type of h-shaped ultrasonic resonator was applied to separate biological particles in [12]. Another study was performed on yeast cells and the red blood cells of rats and guinea pigs in a standing ultrasonic wave in order to identify the empirical boundary conditions of their concentration and separation [13]. An innovative acoustic filter, created using a multilayered 
piezoelectric resonator for the purpose of retaining mammalian cells in cell culture fermentations, was presented in [14].

The work in [15] describes the method applied to biological and non-biological particles in suspension. The mathematical model of blood flow and pressure has been presented in $[16,17]$. Adequate cellular biophysical data can be extracted without invasion due to ultrasound scattering, which allows tissue characterization and determination of red blood cell aggregation $[18,19]$. Some years ago, power ultrasound was considered too expensive for water treatment in the industry [20]. De-watering of bio-sludge (consisting of water, biomass and extracellular polymeric substances) is a complicated process [21]. Ultrafiltration, microfiltration, high performance tangential flow filtration, membrane chromatography and an electrophoretic membrane contactor have become extremely important when dealing with biotechnological products and their separation and purification [22]. In addition, a cell separation device that uses competing electrostatic and acoustic radiation forces to separate cells is presented in [23].

The novelty of this work lies in offering a method for faster purification of larger volumes of microparticles from the biological suspension. It is based on directing ultrasonically-excited heavier suspension particles towards the node areas of standing acoustic pressure waves.

\section{Materials and Methods}

\subsection{Simulation Procedure}

Several biotechnological applications of particle trapping and separation have been reported where ultrasound resonances in microchannels were used to create acoustic fields, giving rise to acoustic radiation forces on suspended particles. Such an acoustophoretic phenomenon creates pressure fluctuations in a fluid medium of moving particles. As the diameter of the particles is much smaller than half the wavelength of the acoustic standing wave, these forces act mainly in one direction, and the particles move towards either a pressure node or pressure anti-node. However, the use of a microchannel produces a very small amount of particles and is therefore useful only in laboratory tests.

Acoustic radiation force is an important nonlinear force exerted by acoustic fields on particles. The acoustic radiation force $F_{r a d}$ on the particle can be calculated as the time averaged second-order forces acting on a fixed surface $\partial \Omega$ in the inviscid bulk, encompassing the particle. For inviscid fluids, vector $F_{\text {rad }}$ is the sum of the time-averaged second-order nonlinear acoustic pressure field $\left\langle p_{2}\right\rangle$ and momentum flux tensor $\rho_{0}\left\langle v_{1} v_{1}\right\rangle$ [24],

$$
\begin{aligned}
& F_{\text {rad }}=-\int_{\partial \Omega} \operatorname{dr}\left\{\left\langle p_{2}\right\rangle n+\rho_{0}\left\langle\left(n \cdot v_{1}\right) v_{1}\right\rangle\right\}= \\
& -\int_{\partial \Omega} \operatorname{dr}\left\{\left[\frac{\kappa_{0}}{2}\left\langle p_{1}^{2}\right\rangle-\frac{\rho_{0}}{2}\left\langle v_{1}^{2}\right\rangle\right] n+\rho_{0}\left\langle\left(n \cdot v_{1}\right)\right\rangle v_{1}\right\}
\end{aligned}
$$

where $\rho_{0}$ is the fluid density, $v_{1}$ is the first-order acoustic velocity field, $p_{1}$ is the first order linear pressure field, $\kappa_{0}$ is the explicit expression for the compressibility and $n$ is the normal vector.

The radiation force acting on a small particle placed in a standing wave is a gradient force of the potential function $U_{\text {rad }}[25]$ :

$$
\begin{gathered}
F_{\text {rad }}=-\nabla U_{\text {rad }}, \\
U_{\text {rad }}=V_{p}\left[f_{1} \frac{1}{2 \rho_{0} c^{2}}\left\langle p^{2}\right\rangle-f_{2} \frac{3}{4} \rho_{0}\left\langle v_{1}^{2}\right\rangle\right], \\
f_{1}=1-\frac{K}{K_{p}}, \quad f_{2}=\frac{2\left(\rho_{p}-\rho_{0}\right)}{2 \rho_{p}+\rho_{0}} .
\end{gathered}
$$

where $\rho_{p}$ is the particle density, $c$ is the speed of sound and $f_{1}$ is real-valued and depends only on the compressibility ratio between the particle and the fluid. 
The dipole scattering coefficient $f_{2}$ is related to the translational motion of the particle and depends on the viscosity of the fluid; $K$ is the bulk modulus of the fluid; $K_{p}$ is the bulk modulus of the particle; and $V_{p}$ is the volume of the particle. The acoustic radiation force on a compressible, spherical, micrometer-sized particle of radius $r=5 \times 10^{-6} \mathrm{~m}$ suspended in a viscous fluid in an ultrasound field of wavelength $\lambda=4.5 \times 10^{-3} \mathrm{~m}$ at room temperature was analyzed, thus $r<<$. The numerical model contained a piezoelectric actuator cylinder with an internal radius of $R=5.5 \times 10^{-3} \mathrm{~m}$ and a direct piezoelectric effect, which was electrically excited by the harmonic law at frequencies $f_{0}=350 \mathrm{kHz}$. The cylinder was filled with a fluid, and by exciting the piezoelectric cylinder at a certain frequency, the standing waves were exposed in it. The modeling procedure was composed of two stages: in the first stage, the particles in a water suspension were simulated; in the second stage, biological suspension properties were attributed to the fluid. Particle parameters were the same in both cases. A 2D model of the system was studied. The model uses the following physical quantities: speed of sound in water: $c=1.57 \times 10^{3} \mathrm{~m} / \mathrm{s}$; the speed of sound in biological suspension: $c=1.48 \times 10^{3} \mathrm{~m} / \mathrm{s}$; $\rho_{p}=4 \times 10^{3} \mathrm{~kg} / \mathrm{m}^{3}$ is the particle density; $K_{p}=2.2 \mathrm{GPa}$ is a bulk modulus of the particle; and the amplitude of the normal acceleration of the transducer is $a_{0}=7.5 \times 10^{6} \mathrm{~m} / \mathrm{s}^{2}$.

\subsection{Experimental Validation Procedure}

To validate the results of the modeling, experiments with a piezo-driven ultrasonic actuator for fluid sonication have been conducted. A prototype actuator was fabricated from off-the-shelf tube-shaped (with dimensions of $\varnothing 19 \times \varnothing 16 \times 28 \mathrm{~mm}$ ) piezoceramic material type PZT-4 (Morgan Advanced Materials Inc., Windsor, UK). This material is ideally suited for ultrasonic radiation applications and is capable of producing large mechanical drive amplitudes while maintaining low mechanical and dielectric losses. The PZT-4 material properties are given in Table 1.

Table 1. Physical properties of PZT-4.

\begin{tabular}{cc}
\hline Density $\left(10^{3} \mathrm{~kg} / \mathrm{m}^{3}\right)$ & 7.7 \\
Young's modulus $\left(10^{10} \mathrm{~N} / \mathrm{m}\right)$ & 7.3 \\
Curie point $\left({ }^{\circ} \mathrm{C}\right)$ & 350 \\
Mechanical Q & 1000 \\
Relative dielectric constant $\varepsilon_{33}^{T} / \varepsilon_{0}$ & 1725 \\
Dielectric loss $(1 \mathrm{kHz})$ & 0.4 \\
$k_{31}$ & 0.32 \\
$k_{p}$ & 0.54 \\
$d_{31}\left(10^{-12} \mathrm{~m} / \mathrm{V}\right)$ & -130 \\
\hline
\end{tabular}

To assess the separation process of microparticles suspended in a fluid using ultrasonication and to determine the vibrating deflection shapes and eigenmodes of the piezoceramic tube-shaped actuator, an experimental setup with a Polytec 3D scanning vibrometer (Type PSV-500-3D-HV, Polytec GmbH, Waldbronn, Germany) was developed and is shown in Figure 1a.

The experimental setup consists of the scanning vibrometer (10), the piezoelectric tube-shaped actuator (6) driven by an ultrasonic frequency signal generator (1, Agilent Technologies, Inc., Loveland, CO 80537, USA) and the voltage amplifier (2, P200-FLC Electronics AB, Sippedalsvagen, Sweden). For the fluid flow rate control and transfer to the actuator, a peristaltic pump (4, model NP-1M, LOIP Ltd., Saint-Petersburg, Russia) is used. The container (3) with the fluid is connected to the inlet tubing of the peristaltic pump, and the outlet tubing of the pump is connected to the inlet (5) of the tube-shaped actuator (6). The bottom-end surface of a tube-shaped piezoelectric actuator (dimensions $\varnothing 19 \times \varnothing 16 \times 28 \mathrm{~mm}$ ) is glued with elastic silicone to the ring-shaped surface (outer diameter $\varnothing 17 \mathrm{~mm}$ ) of the designed collector. Sonication of the fluid is carried out by the use of a tube-shaped actuator; an ultrasound standing wave is applied to separate and distribute microparticles in the researched fluid. The separated particles and liquid phase through the fluid collector (9) and are collected in 
the containers (7 and 8 ). The deformation of the piezoelectric tube-shaped actuator and its frequency response are measured using a Polytec 3D scanning vibrometer (10).

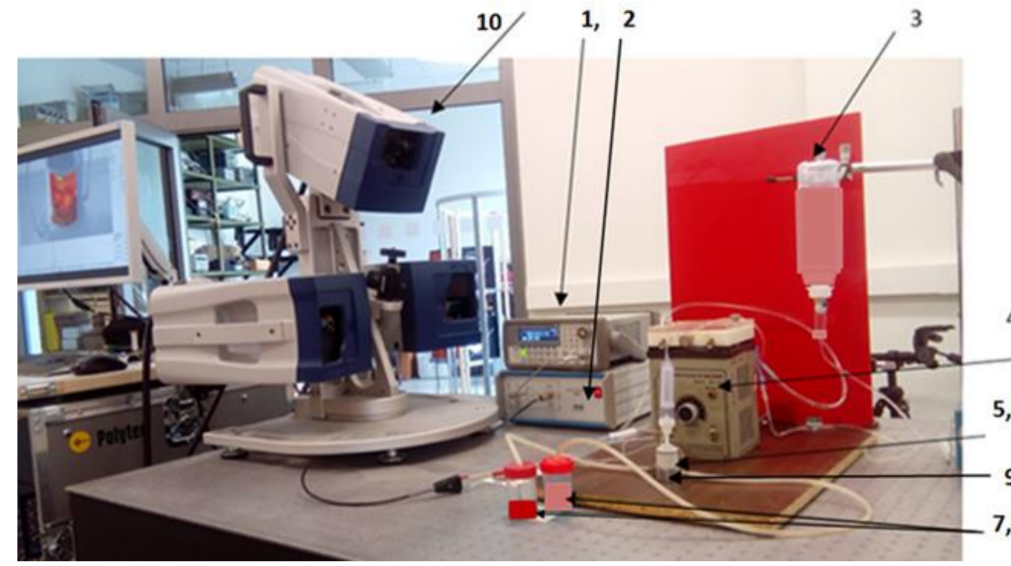

(a)

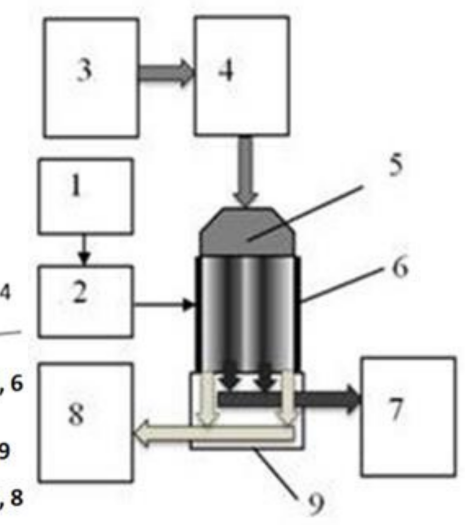

(b)

Figure 1. Photo of the experimental setup (a) and block diagram (b) for the separation of microparticles suspended in a fluid: ultrasonic frequency signal generator (Agilent 33220A) (1), voltage amplifier P200 (FLC Electronics) (2), container of the fluid (3), peristaltic pump (Model NP-1M) (4), inlet of the actuator (5), piezoelectric tube-shaped actuator (6), containers for the microparticles phase and the fluid phase (7 and 8), collector of the fluid (9) and Polytec 3D scanner-vibrometer (10).

A block diagram of the experimental setup is presented in Figure 1b. It explains the direction of fluid movement in the system for the separation of microparticles. In the suspension container (3), suspensions with microparticles are stirred and transferred to the peristaltic pump (4), which regulates the flow rate of the fluid before entering the actuator's inlet (5) and the separation chamber of the piezoelectric tube-shaped actuator (6). An ultrasonic standing wave sonication of the fluid in the flow was established by the piezoelectric actuator, driven by the signal generator (1) and the voltage amplifier (2). After sonication, the suspended microparticles were separated into an enriched and cleared phase by the collector (9), and then, they were transported into separate containers (7 and 8). The duration of the sonication process in the separation chamber of the tube-shaped actuator was controlled by the flow rate of the peristaltic pump (4). The stable separation pattern of microparticles was formed after 5-10 s and was monitored by the Nikon microscope Eclipse LV100 (Nikon Corp., Tokyo, Japan) with a CMOS camera INFINITY1-1C (Lumenera Corporation, Capella Court, Ottawa, ON, Canada).

\section{Results}

\subsection{Simulation Results}

The Comsol Multiphysics software platform (Comsol, Inc., Burlington, MA, USA) was used for simulating the physics-based pressure acoustics problem in the frequency domain. The boundary of the acoustic fluid pressure domain was excited by normal acceleration of a piezoelectric actuator cylinder element. The pressure field of the standing wave in the section of the piezoceramic tube-shaped actuator perpendicular to its axis is shown in Figure 2. This 3D image is tied to the acoustic pressure distribution according to the scale given in the color palette column. 


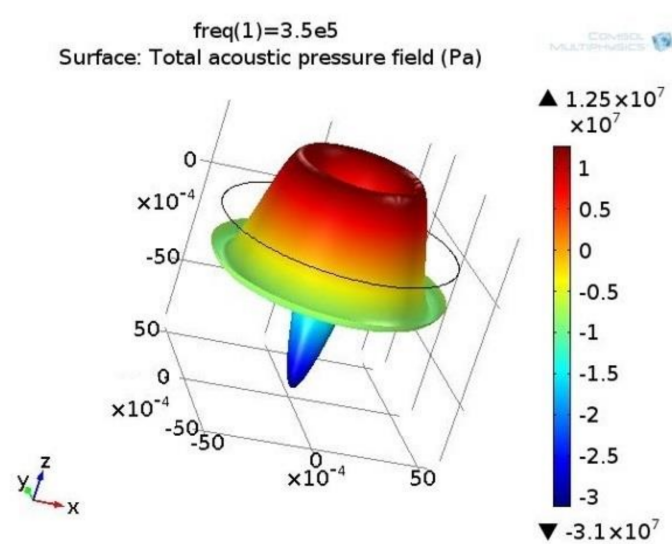

(a)

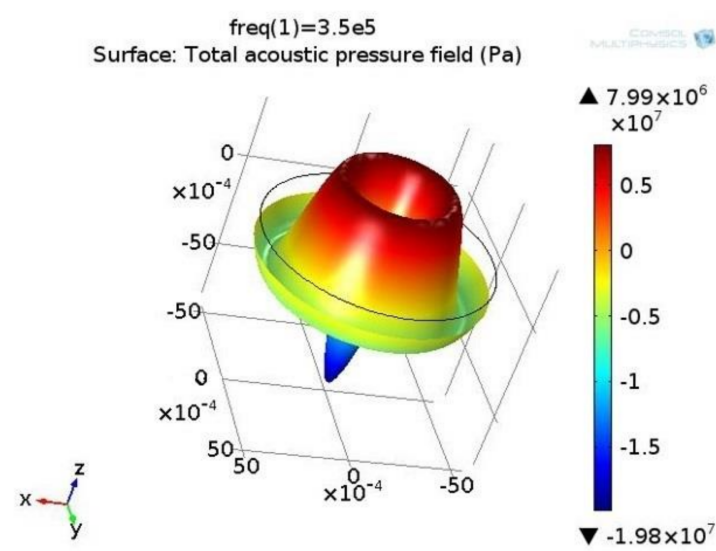

(b)

Figure 2. Pressure field ( $\mathrm{Pa}$ ) of the standing wave in (a) biological and (b) water suspension. Excitation frequency: $350 \mathrm{kHz}$. The high pressure area is represented in the red and blue areas of low pressure. The values of high and low pressure reach several $\mathrm{kPa}$, but in opposite directions.

The sound pressure level is presented in Figure 3.

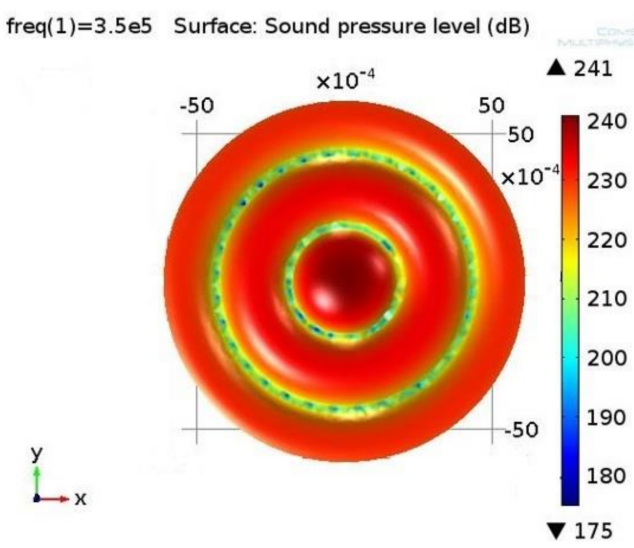

(a)

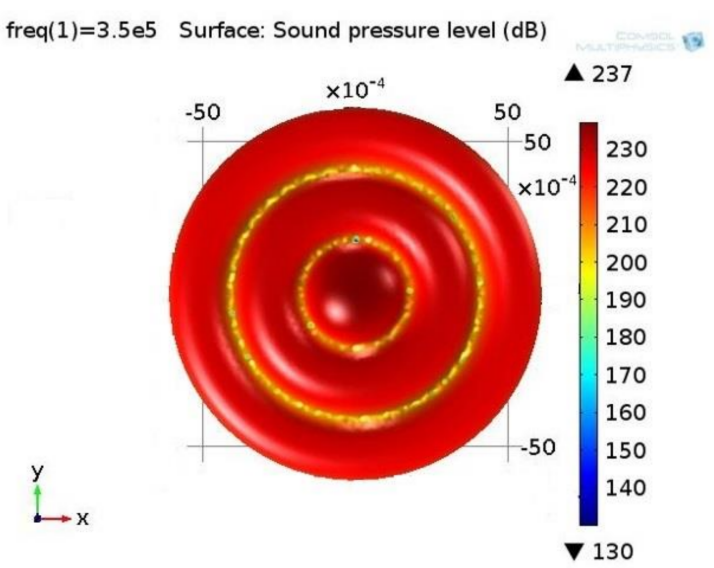

(b)

Figure 3. Sound pressure level $(\mathrm{dB})$ in (a) biological and (b) water suspension. Excitation frequency: $350 \mathrm{kHz}$. The sound pressure field is analogous in both fluids, i.e., there are three high pressure areas and two low pressure areas in between them. However, the low pressure areas differ in different fluids: in the case of a biological suspension, they are wider in comparison to those of the water suspension. This is caused by the different parameter values of the fluids.

The next step is to solve the equation of particle motion in the acoustic field, where the solution determines the position of the particles in the field at the moment of time $t_{i}$. At the initial time $t_{0}$, the particles are uniformly distributed in the acoustic field. Under excitation of the actuator, the ultrasonic standing wave begins to move and concentrate in those areas of the acoustic pressure field where the sound pressure is the smallest. The distribution of the particles in the acoustic field at different points in time is shown in Figure 4 for the biological suspension. The color of the particles represents the speed at the moment of time $t_{i}$, which is calculated by integrating the equations of motion. 


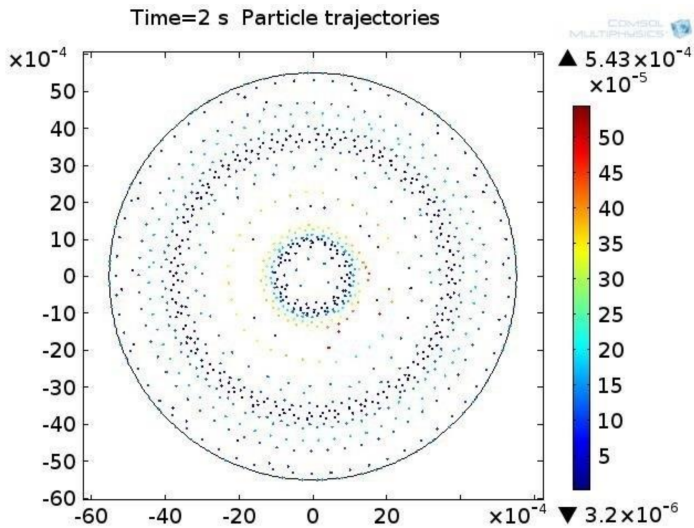

(a)

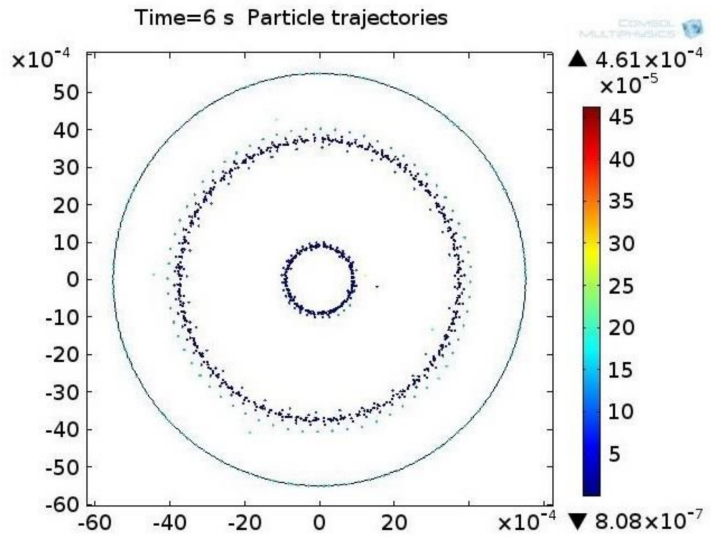

(c)

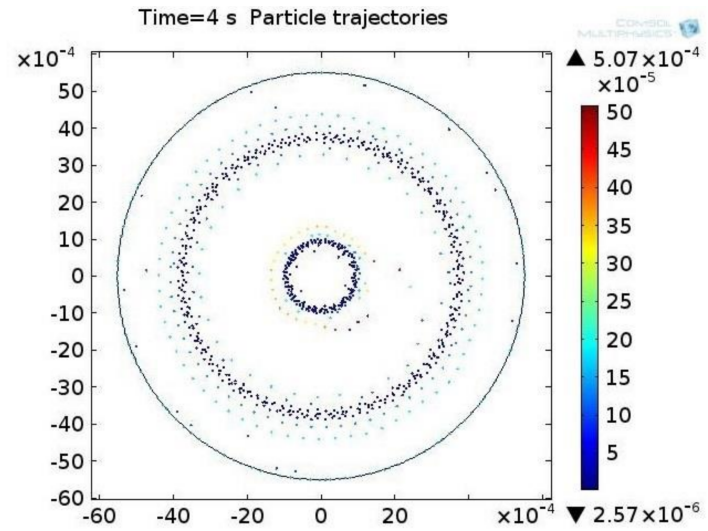

(b)

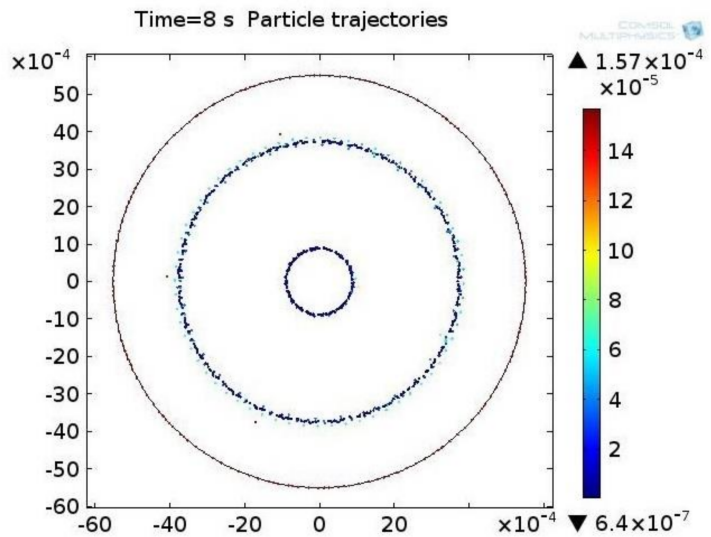

(d)

Figure 4. The distribution of particles in the acoustic pressure field in the biological suspension at different times $t_{i}$ : (a) $2 \mathrm{~s}$, (b) $4 \mathrm{~s}$, (c) $6 \mathrm{~s}$, (d) $8 \mathrm{~s}$.

The distribution of the particles in the acoustic field at different points in time is shown in Figure 5 for the water suspension.

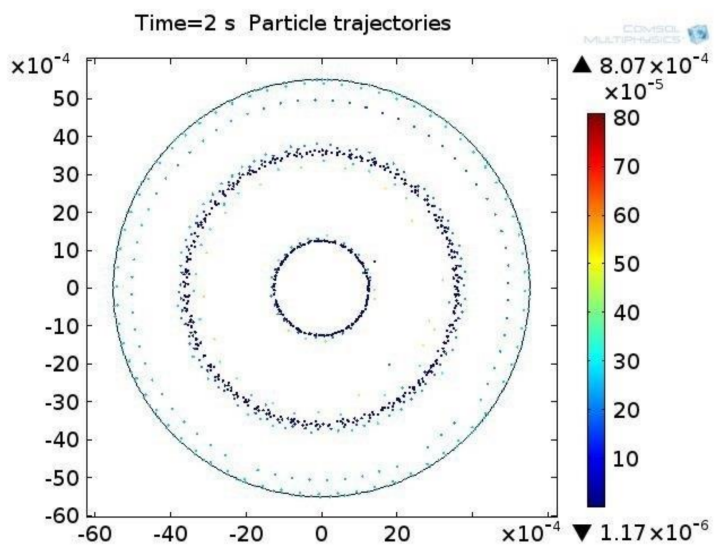

(a)

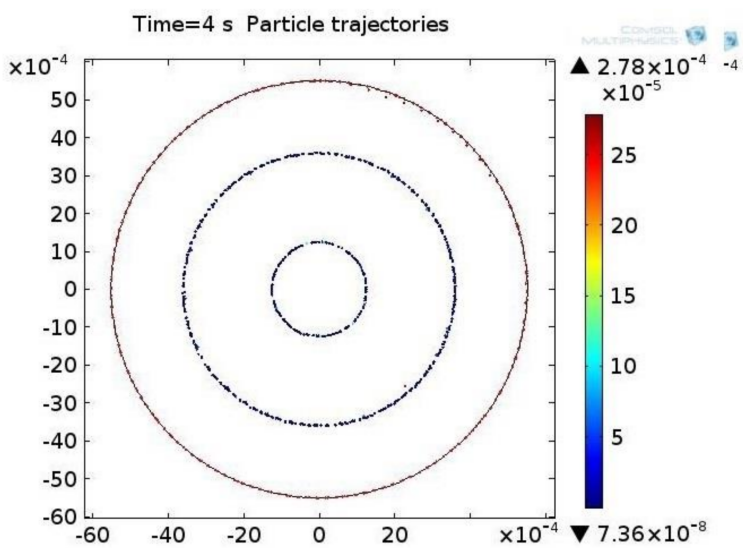

(b)

Figure 5. The distribution of particles in the acoustic pressure field in the water suspension at different times $t_{i}$ : (a) $2 \mathrm{~s}$, (b) $4 \mathrm{~s}$. 


\subsection{Experimental Results}

In the experiments, microparticles in the water and biological suspensions, which correspond to human blood in their viscosity, density and acoustic velocity parameters, were used. As a microparticle phase in the suspension, the material of zeolite with color pigments was used (made by SIGMA-ALDRICH, 3050 Spruce Street, Saint Louis, MO 63103, USA). The microparticle dimensions were $5 \mu \mathrm{m}$.

Vibrating deflection shapes and eigenmodes of the tube-shaped piezoelectric actuator were determined by a Polytec 3D-scanning vibrometer. An outer surface of the tubular actuator was virtually segmented, and each segment was 3D-scanned. A rectangular segment on the piezoelectric actuator outer surface (Figure 6a) was taken perpendicular to the laser beam considering that the symmetrical deflection shapes and eigenmodes detected will help with acoustic and structural dynamics analysis. Figure 6a shows the scanned segment and the grid of the measurement points. To minimize the terrestrial gravity influence on a microparticle separation process, the tubular actuator was positioned vertically. To determine the resonance frequency of the researched actuator suitable for the microparticle separation, the frequency spectrum was obtained in an operational frequency range from $100-650 \mathrm{kHz}$.

Figure 6 shows the deformations of the scanned surface of the actuator. In Figure 6, a segment of the outer surface at the operational frequency of $345 \mathrm{kHz}$ with different fluids is indicated.

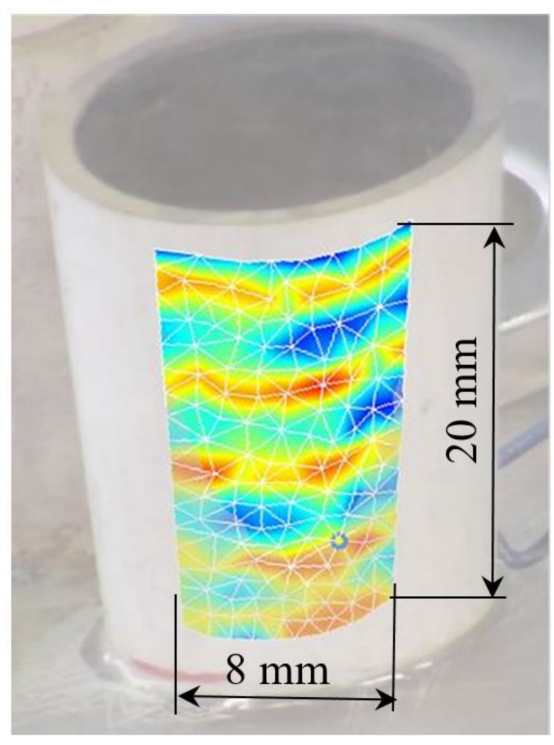

(a)

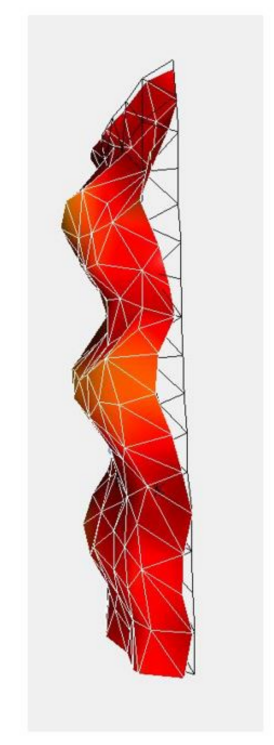

(b)

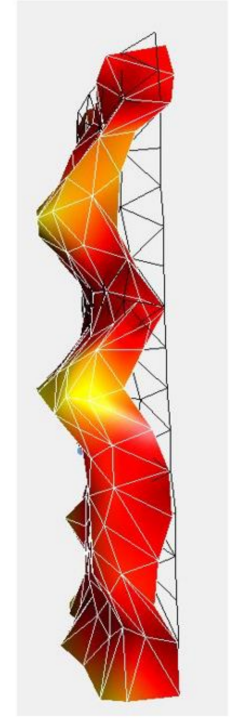

(c)

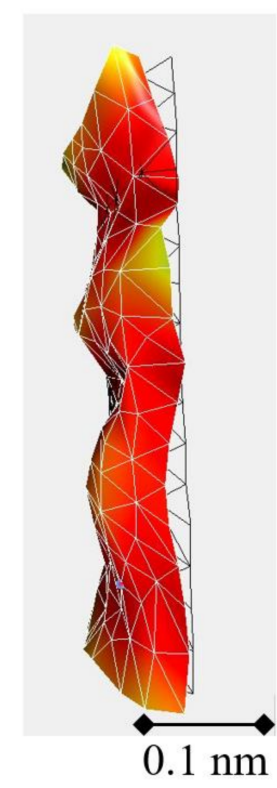

(d)

Figure 6. Photo of the tube-shaped prototype actuator and a segment of its surface (a), which is 3D-scanned to determine vibrating deflection shapes and eigenmodes of the actuator $(\mathbf{b}-\mathbf{d})$.

Measurements were taken for the actuator without a suspension (Figure 6b), with a water-based suspension (Figure 6c) and with a biological suspension-synthetic viscous liquid (Figure 6d). As can be seen from the results, deformations of the actuator with and without the viscous fluid are similar. This is due to viscous damping and fluid inertia.

The frequency spectra of the actuator with the fluid was measured with a 3D scanner (Figure 7) and confirms the simulation results. 


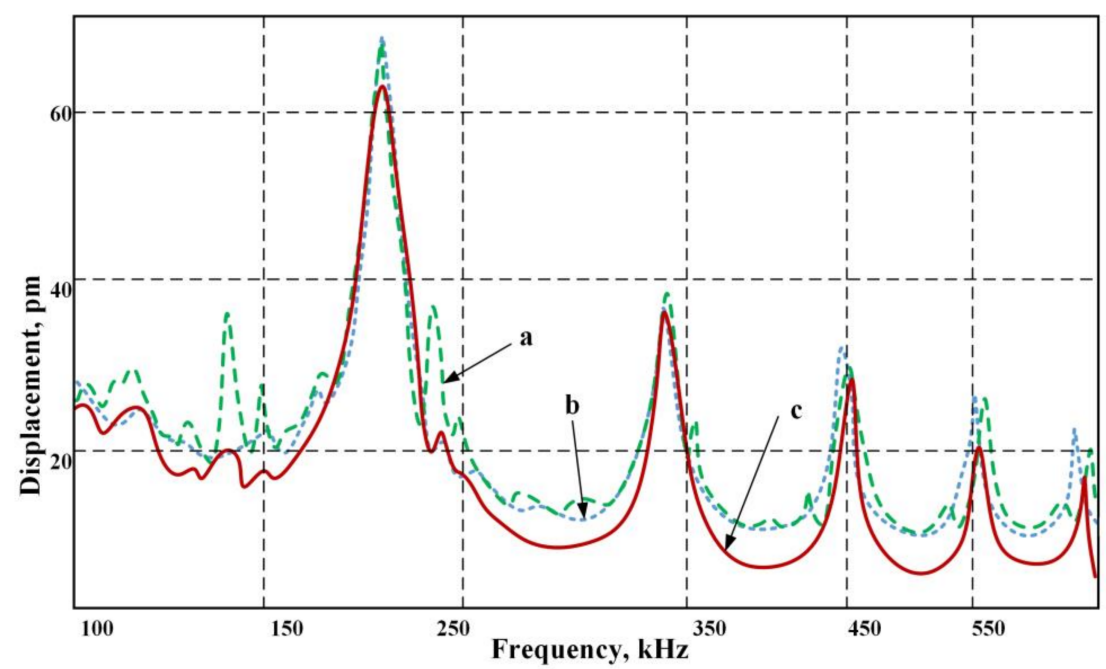

Figure 7. Frequency average spectrum of the actuator scanned surface obtained by measurement via a 3D scanner: (a) without fluid; (b) with the water suspension; (c) with the biological suspension-viscous fluid.

A stable resonance frequency of $345 \mathrm{kHz}$ allowed us to obtain a constant suspension flow separated into fractions. At a frequency of $202 \mathrm{kHz}$, the amplitude of deformation is the greatest, but the study showed flow instability that led to the suspension mixing rather than its separation. Therefore, we had to give up the greater amplitude in order to continually collect particles and move them to the desired trajectory of the ultrasonic signal. Here, (a) denotes the deformation in the fluid without suspension, (b) stands for a signal for a water suspension and (c) describes the operation when using a biological suspension-viscous fluid. As can be seen from the chart, with a viscous flow, there is a certain quenching strain on non-resonant frequencies.

The separation of the microparticles from the suspension in the piezoelectric cylinder under the influence of the standing ultrasonic wave is shown in Figure 8. For comparison, data are given for a frequency of $202 \mathrm{kHz}$ (Figure 8a) and for a frequency of $345 \mathrm{kHz}$ (Figure $8 \mathrm{~b}$ ). The time spent on the separation of the suspension flow into fractions was four seconds for a water-based suspension and eight seconds for a biological suspension-viscous fluid.

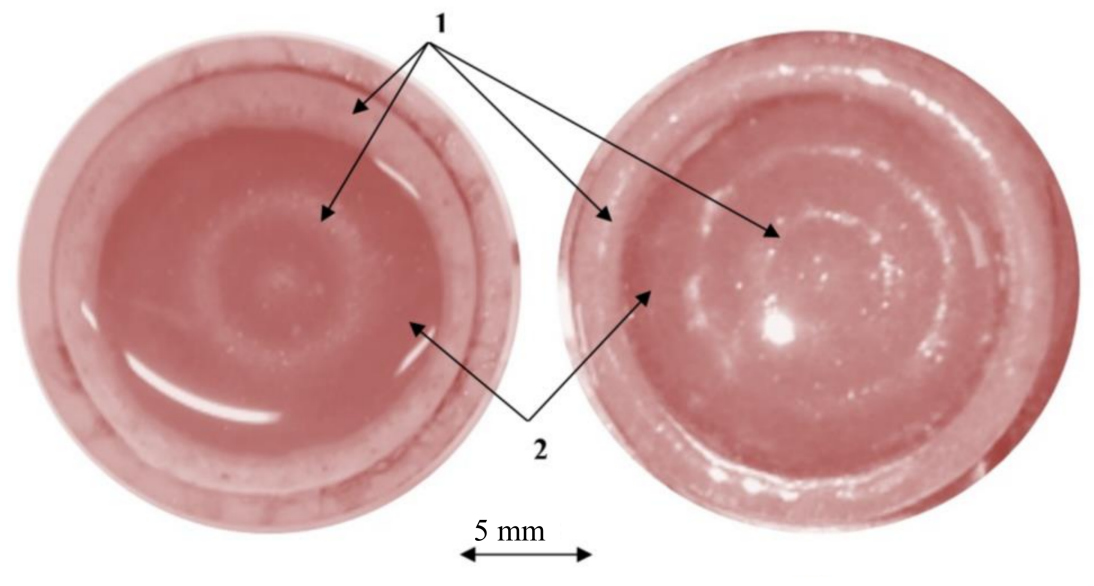

(a)

(b)

Figure 8. The distribution of the particles in the cylinder with the suspension under the influence of a standing ultrasonic wave: (a) excitation frequency of $202 \mathrm{kHz}$; (b) excitation frequency of $345 \mathrm{kHz}$; the microparticles distribution in standing wave nodal (1) and anti-nodal (2) circles. 
For the extraction of microparticles from the biological suspension, a special collector was designed and manufactured (Figure 9). The distribution of the channels of this collector are located in the circles, the centerline diameters of which coincide with the diameters of the acoustic standing wave nodal circles or low pressure areas, in which the solid microparticles precipitate ( 4 and $14 \mathrm{~mm}$, respectively). After eight seconds of ultrasonic treatment in the piezoelectric cylinder, the enriched microparticle mixture was accumulated via the channels of the collector.

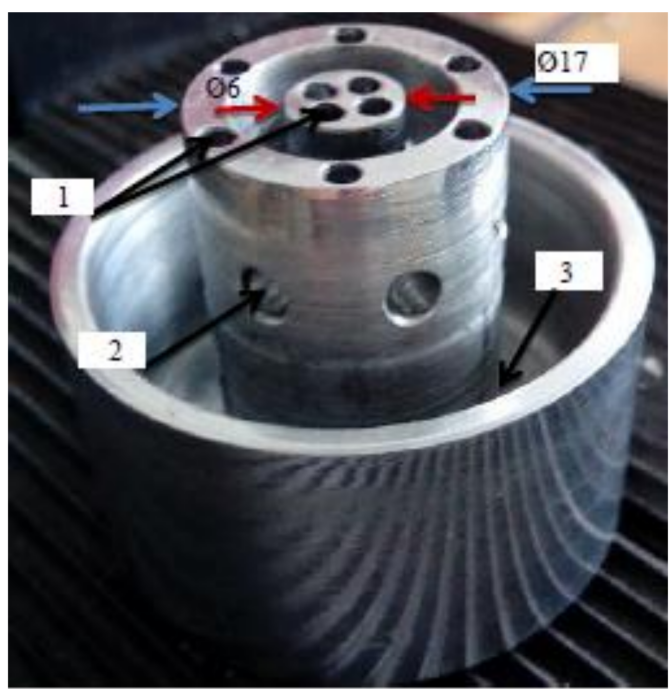

Figure 9. Photo of the collector. 1: openings for collection of the microparticle phase; 2: openings for collection of the suspension phase; 3 : volume of the suspension phase.

To determine the effectiveness of the fluid purification, two separate samples were examined under a microscope. The results are shown in Figure 10. The image in (a) depicts a sample of microparticles in the biological suspension before and (b) after the separation procedure. The quantity of microparticles is increased by 6.5-times.

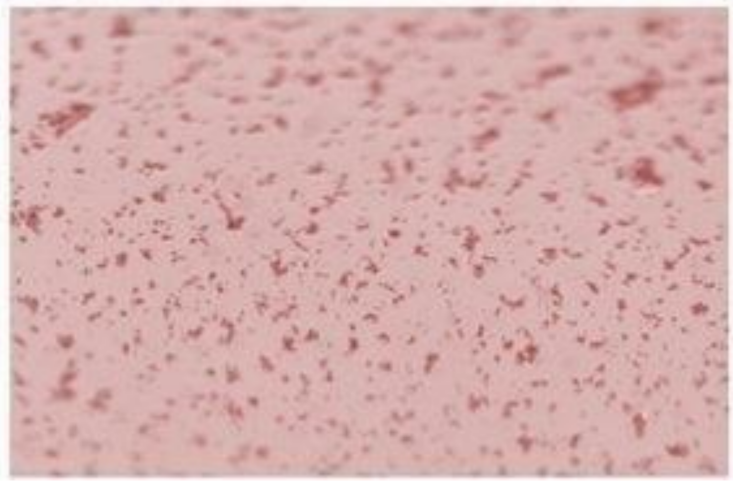

(a)

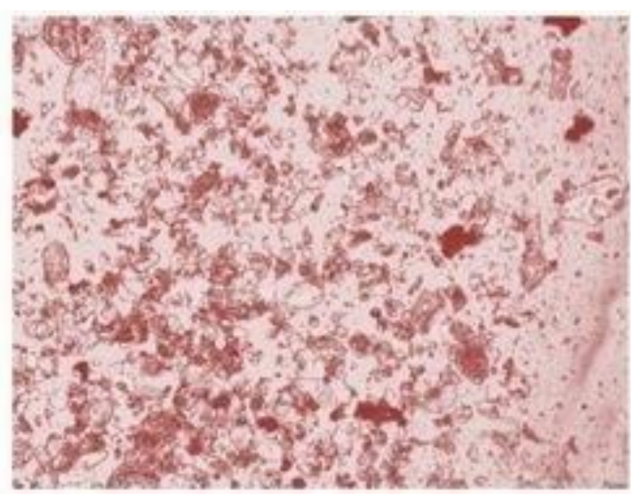

(b)

Figure 10. Microscopic view of the biological suspension samples before (a) and after (b) the purification procedure.

\section{Discussion}

For rapid microparticle purification to be realized, some conventional methods were analyzed, and it was concluded that the most suitable technique for this purpose is the ultrasonic method. However, it is well known that the ultrasonic purification method in microchannels is not suitable 
for larger amounts of fluid. Consequently, the method of suspension treatment in a piezoelectric cylinder has been proposed. It was found that the distribution of both biological and water suspension particles in the acoustic pressure field is the same, yet the velocity of particle convergence to the low-level pressure field is different. When the fluid is water, the particles get into the low sound pressure field in $4 \mathrm{~s}$, whereas in the case of the biological suspension, it takes twice the time, i.e., $8 \mathrm{~s}$. Another difference between the fluids under investigation is the particle distribution in low sound pressure fields. Concentration areas of biological suspension particles at the end of the process, i.e., when the fluid becomes stationary, are broader in comparison to concentration areas obtained using water as a fluid (see Figure 3). Both peculiarities may be explained by different values of the analyzed fluid properties. Finally, the third difference lies in the geometrical discrepancy of the small radius low sound pressure level areas; in the case of the biological suspension, the area radius is smaller by $30 \%$ in comparison to that of water. This fact should be taken into consideration when separating biological suspensions of various viscosities. Consideration of this effect enables us to control the particle distribution in the desired areas of the acoustic field. This results in a quick and reliable particle concentration in the desired acoustic field areas (by changing the characteristics of vibrations generated by the actuator). This is a technological approach for the removal of particles from suspensions.

As only a physical experiment with an elaborated purification device could confirm the appropriateness of a mathematical model, the experimental setup was assembled in order to ensure precise measurements. Two types of suspensions were considered that included particles of the diameter of erythrocytes: biological and water-based. We choose these types of suspensions to demonstrate, by experiment, the universality and validity of the proposed purification technology. Two main parameters of the particles that form a nodal circle are related to the fluid acoustical excitation frequency and the constructive dimensions of the piezoelectric actuator. Since the number of nodal circles or low pressure areas in which the solid microparticles precipitate is increased at higher frequencies and was concentrated at a frequency of $345 \mathrm{kHz}$, the lower frequency of $202 \mathrm{kHz}$ was abandoned in favor of stable and efficient acoustic forces produced at a frequency of $345 \mathrm{kHz}$. The diameters of microparticle concentration circles coincide in both the simulation and experimentation cases.

As was shown experimentally and theoretically, the diameters of particle aggregation circles for both suspensions were identical, whereas the period of circle formation differed two-fold. This period (from four to eight seconds) could be considered very short compared to other known methods of purification [26]. Despite the fact that the cells in biological suspensions are very susceptible to the influence of ultrasonic signals, our experience has shown that the frequency of $345 \mathrm{kHz}$ is safe for cell membranes [27]. Whereas a theory should make predictions, a good theory's predictions should be supported by the results of experiments. Good correlation between model and experimental data of piezoceramic tube vibrations has been observed in [28]. In our case, the matching of the simulation $(350 \mathrm{kHz})$ and experimentally-obtained $(345 \mathrm{kHz})$ ultrasonic excitation frequencies, as well as the coincidence of the diameters of microparticle concentration circles in both the simulation and experimentation cases suggest that the mathematical model imitates the real microparticle purification process. The simulation results show that particle density does not affect the acoustic pressure, because it is created by an external effect; in this case, it is the reverse piezo effect. However, the density of the particles influences the separation time interval. This time interval is particularly increased when the fluid and the particle density ratio are approaching unity. As the fluid and the particle density ratio are very important, from an engineering point of view, this ratio directly affects the piezo cylinder length. In our case, the length of piezo cylinder and the flow rate of the fluid were sufficient for the microparticle separation time interval to be equal to $8 \mathrm{~s}$. In theory, if the fluid and the microparticle density are close, the purification time increases considerably, thus requiring a greater length for the separator. For example, to evaluate microparticles separation efficiency in $\mathrm{L} / \mathrm{h}$, as estimated in the article [29], the flow rate should be $0.278 \mathrm{~mL} / \mathrm{s}$. In our case with the volume of the piezoceramic tube-shaped actuator, we get that the separator's performance as over $2 \mathrm{~L} / \mathrm{h}$. The proposed method is more than 2.0-times faster than the separation of particles in the microchannel. In contrast to the separation of the particles 
in the microchannel, the amount of fluid treated in the piezoceramic tube-shaped actuator depends on its volume, which can be selected according to the amount of fluid to be treated.

\section{Conclusions}

A straightforward method for the separation of microparticles is proposed. A finite element model is implemented to simulate the radiated fields of a cylindrical ultrasonic actuator with its volume filled by a suspension. Microparticle concentration takes place in the nodal circles area of standing ultrasonic waves. It was found that the distribution character of different types of microfluid particles in the acoustic pressure field is the same, but their distribution speed depends on the suspension viscosity. As the amount of microfluid depends on the construction of a piezoelectric actuator, larger volumes of microparticles could be purified. The proposed method is simple to realize, does not require large investments and the elaborated device is transportable. The results obtained here are a prerequisite for the development of an experimental prototype for microparticle separation. With these results, it can be concluded that the separation of different sizes and densities of microparticles from the various viscosity suspensions is possible.

Acknowledgments: This work was funded by a grant (No. SEN-10/15) from the Research Council of Lithuania. Project acronym: "CaSpine".

Author Contributions: Vytautas Ostasevicius wrote the paper. Vytautas Jurenas and Ievgeniia Golinka conceived, designed and performed the experiments. Rimvydas Gaidys and Algiment Aleksa contributed to the analyses of the data.

Conflicts of Interest: The authors declare no conflict of interest. The founding sponsors had no role in the design of the study; in the collection, analyses or interpretation of data; in the writing of the manuscript; nor in the decision to publish the results.

\section{References}

1. Wloch, A.; Czyz, H.; Jasinski, T. Ultrasonic methods of the cells separation in human blood. Acta Phys. Pol. A 2015, 128, 234-236. [CrossRef]

2. Lei, J.; Glynne-Jones, P.; Hill, M. Acoustic streaming in the transducer plane in ultrasonic particle manipulation devices. Lab Chip 2013, 13, 2133-2143. [CrossRef] [PubMed]

3. Mohamed, M.A.; Fikry, N.M.; Mohamed, M.M. Experimental investigation of ultrasonic trapping of bioparticles using parallel plane cavity. Int. J. Biomed. Eng. Sci. 2014, 1, 21-34.

4. Setayeshgar, A.; Lipsett, M.G.; Koch, C.R.; Nobes, D.S. Measurement of particle dynamics in a coherent acoustic field. In Proceedings of the 10th International Symposium on Particle Image Velocimetry, Delft, The Netherlands, 1-3 July 2013; p. 6.

5. Westerweel, J. Fundamentals of digital particle image velocimetry. Meas. Sci. Technol. 1997, 8, 1379-1392. [CrossRef]

6. Devendran, C.; Gralinski, I.; Neild, A. Separation of particles using acoustic streaming and radiation forces in an open microfluidic channel. Microfluid. Nanofluid. 2014, 17, 879-890. [CrossRef]

7. Haddadi, B.; Fathipour, M. Numerical analysis of 3D model of the SSAW separator system. Int. J. Comput. Appl. 2016, 141, 7-12. [CrossRef]

8. Kapishnikov, S.; Kantsler, V.; Steinberg, V. Continuous particle size separation and size sorting using ultrasound in a microchannel. J. Stat. Mech. Theory Exp. 2006, P01012. [CrossRef]

9. Laurell, T.; Petersson, F.; Nilsson, A. Chip integrated strategies for acoustic separation and manipulation of cells and particles. Chem. Soc. Rev. 2007, 36, 492-506. [CrossRef] [PubMed]

10. Oberti, S.; Möller, D.; Neild, A.; Duala, J.; Beyelerb, F.; Nelson, B.J.; Gutmann, S. Strategies for single particle manipulation using acoustic and flow fields. Ultrasonics 2010, 50, 247-257. [CrossRef] [PubMed]

11. Petersson, F.; Nilsson, A.; Holm, C.; Jonsson, H.; Laurell, T. Separation of lipids from blood utilizing ultrasonic standing waves in microfluidic channels. R. Soc. Chem. J. Anal. 2004, 129, 938-943. [CrossRef] [PubMed]

12. Böhm, H.; Briarty, L.G.; Lowe, K.C.; Power, J.B.; Benes, E.; Davey, M.R. Application of a novel h-shaped ultrasonic particle separator under microgravity conditions. In Proceedings of the Forum Acusticum 2002, Special Session PHA-01: Acoustics of Dispersed Particulate Matter, Sevilla, Spain, 16-20 September 2002. 
13. Sadikova, D.G.; Pashovkin, T.N. Cell concentration and separation in the field of a standing ultrasonic wave for medicine and biotechnology. Open J. Biophys. 2013, 3, 70-75. [CrossRef]

14. Grösch, M.; Burger, W.; Handl, B.; Doblhoff-Dier, O.; Gaida, T.; Schmatz, C. Ultrasonic separation of suspended particles-Part III. Acta Acust. United Acust. 1998, 84, 815-822.

15. Riera-Franco de Sarabia, E.; Gallego-Juárez, J.A.; Rodríguez-Corral, G.; Elvira-Segura, L.; González-Gómez, I. Application of high-power ultrasound to enhance fluid/solid particle separation processes. Ultrasonics 2000, 38, 642-646. [CrossRef]

16. Andrade, M.A.B.; Buiochi, F.; Adamowski, J.C. Particle manipulation by ultrasonic progressive waves. Phys. Proc. 2010, 3, 283-288. [CrossRef]

17. Rahman, S.; Haque, A. Mathematical modeling of blood flow. In Proceedings of the 2012 International Conference on Informatics, Electronics \& Vision (ICIEV), Dhaka, Bangladesh, 18-19 May 2012; pp. 672-676.

18. Fontaine, I.; Savery, D.; Cloutier, G. Simulation of ultrasound backscattering by red cell aggregates: Effect of shear rate and anisotropy. Biophys. J. 2002, 82, 1696-1710. [CrossRef]

19. Liu, Y.; Liu, W.K. Rheology of red blood cell aggregation by computer simulation. J. Comput. Phys. 2006, 220, 139-154. [CrossRef]

20. Mason, T.J.; Joyce, E.; Phull, S.S.; Lorimer, J.P. Potential uses of ultrasound in the biological decontamination of water. Ultrason. Sonochem. 2003, 10, 319-323. [CrossRef]

21. Yin, X.; Han, P.; Lu, X.; Wang, Y. A review on the dewaterability of bio-sludge and ultrasound pretreatment. Ultrason. Sonochem. 2004, 11, 337-348. [CrossRef] [PubMed]

22. Saxena, A.; Tripathi, B.P.; Kumar, M.; Shahi, V.K. Membrane-based techniques for the separation and purification of proteins: An overview. Adv. Colloid Interface Sci. 2009, 145, 1-22. [CrossRef] [PubMed]

23. Yasuda, K.; Sakamoto, T. Cell separation device using ultrasound and electrophoresis. U.S. Patent 6245207 B112, 18 May 1999.

24. Settnes, M.; Bruus, H. Forces acting on a small particle in an acoustical field in a viscous fluid. Phys. Rev. 2012, E85, 1-12. [CrossRef]

25. Gor'kov, L.P. On the forces acting on a small particle in an acoustical field in an ideal fluid. Sov. Phys. Dokl. $1962,6,773$.

26. Stegmayr, B.G. A survey of blood purification techniques. Transf. Apher. Sci. 2005, 32, 209-220. [CrossRef] [PubMed]

27. Wu, J.; Nyborg, W.L. Ultrasound, cavitation bubbles and their interaction with cells. Adv. Drug Deliv. Rev. 2008, 60, 1103-1116. [CrossRef] [PubMed]

28. Lewis, R.W.C.; Bowen, C.R.; Dent, A.C.E.; Jonas, K. Finite element and experimental analysis of the vibration response of radially poled piezoceramic cylinders. Ferroelectrics 2010, 389, 95-106. [CrossRef]

29. Adams, J.D.; Ebbesen, C.L.; Brankob, R.; Yang, A.H.J.; Soh, H.T.; Bruus, H. High-throughput, temperature-controlled microchannel acoustophoresis device made with rapid prototyping. J. Micromech. Microeng. 2012, 22, 1-4. [CrossRef]

(C) 2018 by the authors. Licensee MDPI, Basel, Switzerland. This article is an open access article distributed under the terms and conditions of the Creative Commons Attribution (CC BY) license (http://creativecommons.org/licenses/by/4.0/). 